\title{
LncRNA TUG1 promotes tumor growth and metastasis of esophageal squamous cell carcinoma by regulating XBP1 via competitively binding to miR-498
}

\author{
G. JIN ${ }^{1}$, Y. YANG 2 , G. TUO 2 , W. WANG 1 , Z. ZHU' \\ ${ }^{1}$ Department of Thoracic Surgery 2, Gansu Provincial Hospital, Lanzhou, China; ${ }^{2}$ Department of Clinical Medicine, Gansu University of Tradi- \\ tional Chinese Medicine, Lanzhou, China \\ *Correspondence: zhuzijiang20191101@163.com
}

Received May 21, 2018 / Accepted July 22, 2019

\begin{abstract}
Esophageal squamous cell carcinoma (ESCC) is a major subtype of esophageal cancer with high mortality. Previous reports suggested that lncRNA taurine upregulated gene 1 (TUG1) functioned as an oncogene in numerous cancers. The purpose of this study was to explore the potential mechanism of TUG1 carcinogenesis in ESCC. The expression of TUG1 and miR-498 was measured by a quantitative real-time polymerase chain reaction (qRT-PCR). Cell proliferation and apoptosis were assessed by 3-(4, 5-dimethyl-2-thiazolyl)-2, 5-diphenyl-2-H-tetrazolium bromide (MTT) assay and flow cytometry. Cell migration and invasion were identified through the transwell assay. The interaction between miR-498 and TUG1 or $\mathrm{X}$-box binding protein 1 (XBP1) was predicted by bioinformatics software starBase and verified by luciferase reporter assay. The expression of XBP1 was quantified by qRT-PCR and western blot analysis. Xenograft tumor mouse model was established to determine the function of TUG1 in vivo. TUG1 was upregulated in ESCC tissues and cells, and its high expression was associated with tumor lymph node metastasis and low cumulative survival. TUG1 knockdown inhibited proliferation, migration, and invasion but promoted apoptosis in ESCC cells. It was confirmed that miR-498 was a target of TUG1, and XBP1 was a target of miR-498. The expression of miR-498 was reduced in ESCC tissues while XBP1 expression was notably enhanced. Mechanism analysis manifested that TUG1 regulated proliferation, apoptosis, migration, and invasion by upregulating XBP1 via targeting miR-498 in vitro. Furthermore, knockdown of TUG1 attenuated tumor growth in vivo. TUG1 accelerated tumorigenesis and metastasis by inducing XBP1 expression through directly targeting miR-498 in ESCC.
\end{abstract}

Key words: esophageal squamous cell carcinoma, TUG1, miR-498, XBP1

Human esophageal cancer is one of the most common malignancies worldwide and is the sixth leading cause of death from cancer [1]. Esophageal cancer is divided into two forms, including esophageal squamous cell carcinoma (ESCC) and adenocarcinoma, with different etiology and pathological features [2]. ESCC is a major subtype of esophageal cancer and accounts for the majority of the cases discovered in Asia with a poorer prognosis than that of adenocarcinoma [2-4]. Recently, despite the progress of therapeutic options has been made, such as surgery, chemotherapy, and radiotherapy, the prognosis of patients with ESCC is still unsatisfactory [5]. Therefore, efforts to search biomarkers associated with the pathogenesis of ESCC are necessary.

Long non-coding RNAs (lncRNAs) represent a class of endogenous RNAs with a transcript length of over 200 nucleotides [6]. They exert their function by regulating gene expression at three levels: epigenetic regulation, transcriptional regulation, and post-transcriptional regulation [7]. The increasing number of evidence suggests that lncRNAs dysregulation is associated with tumor occurrence, metastasis, diagnosis or prognosis by partly acting as oncogenes or tumor suppressors in the development of multiple tumors [8, 9]. LncRNA taurine upregulated gene 1 (TUG1), located on chromosome $22 \mathrm{q} 12.2$, was upregulated with the addition of taurine in developing mouse retinal cells [10]. Presently, some studies reported that TUG1 functioned as an oncogene and was aberrantly expressed in many human cancers, including non-small cell lung cancer (NSCLC) [11], gastric cancer [12], colorectal cancer [13], hepatocellular carcinoma [14], and urothelial carcinoma [15]. These data suggest the crucial role of TUG1 in human cancers. However, the research on the 
role of TUG1 in ESCC and associated functional mechanism is still limited.

Generally, lncRNAs can serve as precursor molecules of microRNAs (miRNAs) for transcription. MicroRNAs (miRNAs), 19-25 nucleotides in length, are a class of important non-coding RNAs that negatively regulate gene expression at the post-transcriptional or translational level [16]. Recent studies claim that miRNAs are involved in numerous biological processes, regulating several signaling pathways by acting as key tumor-suppressor genes in many human cancers [17]. For ESCC, a variety of aberrantly expressed miRNAs had been reported, such as miR-21 [18], miR-146a [19], and miR-203 [20]. miR-498 is located in 19q13.42 and has been found to be a functional modulator in various cancers, including NSCLC [21], ovarian cancer [22], and colorectal cancer [23]. However, the exhaustive function of miR-498 in ESCC remains unclear.

Human X-box binding protein 1 (XBP1), belonging to the basic region/leucine zipper protein family, is an indispensable transcription factor that participates in many signal transduction processes [24]. Previous studies stated that XBP1 was involved in several cancers by conducting cell type- and tissue-specific transcriptional regulatory networks [25]. For example, XBP1 contributed to tumor invasion through upregulating MMP9 expression in ESCC [26]. XBP1 promoted cell invasion by inducing the key epithelial-tomesenchymal transition (EMT) regulator snail expression to activate EMT in breast cancer cells [27]. These reports reveal the irreplaceable role of XBP1 in human cancers. Therefore, the study of XBP1 in ESCC is valuable and practical.

In this study, we detected the expression and potential role of TUG1, explored the interaction between miR-498 and TUG1 or XBP1 as well as their expression in vitro, and investigated the function of TUG1 by xenograft model in vivo. The present study aimed to provide an underlying action mechanism of TUG1 in ESCC.

\section{Patients and methods}

Samples and cell lines. This research was approved by the Gansu Provincial Hospital Ethics Committee. A total of 50 paired ESCC tumor tissues and adjacent normal tissues were collected in Gansu Provincial Hospital. Before clinical surgery, all patients signed informed consent forms. These samples were immediately frozen in liquid nitrogen after removing from bodies and then stored at $-80^{\circ} \mathrm{C}$ condition for the following use.

The human ESCC cell lines (KYSE-150, EC109, KYSE450, TE-10, and KYSE-410) and human normal esophageal squamous epithelial cell line Het-1A were all purchased from BeNa Culture collection (BNCC, Suzhou, China). All cells were maintained in Roswell Park Memorial Institute 1640 (RPMI-1640; Gibco, Grand Island, NY, USA) supplemented with 10\% fetal bovine serum (FBS; Gibco), $100 \mathrm{IU} / \mathrm{ml}$ penicillin sodium (Gibco) and $100 \mu \mathrm{g} / \mathrm{ml}$ strepto- mycin sulfate (Gibco) at $37^{\circ} \mathrm{C}$ in a humidified atmosphere containing $5 \% \mathrm{CO}_{2}$.

Cell transfection. For TUG1 knockdown and overexpression, small interference RNA against TUG1 (si-TUG1), negative control (si-NC), overexpression vector pcDNA3.1 containing TUG1 (TUG1) and pcDNA3.1 empty vector (vector) were all purchased or synthesized by GenePharma (Shanghai, China). For miR-498 overexpression and inhibition, miR-498 mimics, miR-498 inhibition (anti-miR-498), and their corresponding controls (miR-NC and anti-NC) were obtained from RIBOBIO (Guangzhou, China). For XBP1 overexpression, the pcDNA3.1 overexpression vector containing XBP1 (pcDNA-XBP1) and pcDNA3.1 empty vector (pcDNA) were synthesized from GenePharma. All transient transfection with oligonucleotides or plasmids into KYSE-150 and EC109 cells was conducted by Lipofectamine ${ }^{\mathrm{TM}}$ 2000 (Thermo Fisher Scientific, Waltham, MA, USA). Cells were harvested after $48 \mathrm{~h}$ for the following analyses.

Quantitative real-time polymerase chain reaction (qRT-PCR). Total RNA was isolated from ESCC tissues and cells using Trizol reagent (Invitrogen, Carlsbad, CA, USA). Then the RNA was reversely transcribed into complementary DNA (cDNA) using High-Capacity cDNA Reverse Transcription Kits (Thermo Fisher Scientific) for detection of TUG1 and XBP1, or using MicroRNA Reverse Transcription Kit (Thermo Fisher Scientific) for detection of miR-498. Then qRT-PCR was performed using the SYBR Premix Ex TaqII kit (Takara, Tokyo, Japan) with 1 $\mu \mathrm{l}$ cDNA on ABI 7900 system (Applied Biosystems, Foster City, CA, USA) according to the manufacturer's instructions. Fold change of expression was calculated using the $2^{-\Delta \Delta C t}$ method and normalized by glyceraldehyde-3-phosphate dehydrogenase (GAPDH) or U6. The primers were as follows: TUG1, forward: 5'-TAGCAGTTCCCCAATCC TTG-3' and reverse: 5'-CACAAATTCCCATCATTCCC-3'; XBP1, forward: 5'-CCCTCCAGAACATCTCCCCAT-3' and reverse: 5'-ACATGACTGGGTCCAAGTTGT-3'; GAPDH, forward: 5'-CCATTTGCAGTGGCAAAG-3' and reverse: 5'-CACCCCATTTGATGTTAGTG-3'; miR-498, forward: 5'-TTTCAAGCCAGGGGGCGTTTTTC-3' and reverse: 5'-GCTTCAAGCTCTGGAGGTGCTTTTC-3'; U6, forward: 5'-ACCCTGAGAAATACCCTCACAT-3' and reverse: 5'-GACGACTGAGCCCCTGATG-3'.

3-(4, 5-dimethyl-2-thiazolyl)-2, 5-diphenyl-2-H-tetrazolium bromide (MTT) assay. KYSE-150 and EC109 cells were seeded into 96-well plates after transfection. At $24 \mathrm{~h}$, $48 \mathrm{~h}, 72 \mathrm{~h}$ and $96 \mathrm{~h}, 0.5 \mathrm{mg} / \mathrm{ml}$ MTT solution (Sigma-Aldrich, St. Louis, MO, USA) was added into each well and cells were incubated for $4 \mathrm{~h}$. The optical density (OD) value at $490 \mathrm{~nm}$ was measured by a microplate reader (Bio-Rad, Hercules, CA, USA). Five replicates were conducted for each group.

Flow cytometry analysis. Flow cytometry was performed to monitor cell apoptosis by using the Dead Cell Apoptosis Kit (Invitrogen). Briefly, KYSE-150 and EC109 cells $\left(1 \times 10^{6}\right.$ cells $/ \mathrm{ml}$ ) were collected, washed with pre-cooled PBS and 
re-suspended in $1 \times$ Annexin-binding buffer according to the product's protocol. Next, $5 \mu \mathrm{l}$ of fluorescein isothiocyanate (FITC) Annexin V and $1 \mu \mathrm{l}$ of the $100 \mu \mathrm{g} / \mathrm{ml}$ PI working solution were added into each $100 \mu \mathrm{l}$ cell suspension for 15 min incubation. Subsequently, the apoptotic cells were distinguished by using flow cytometer $\mathrm{S}{ }^{\text {tm }}$ Cell Sorter (Bio-Rad).

Transwell assay. Transwell assay was carried out to analyze cell migration and invasion. In brief, KYSE-150 and EC109 cells $\left(3 \times 10^{4}\right.$ cells $)$ were harvested at $48 \mathrm{~h}$ post-transfection. For migration assay, cells were placed in $100 \mu \mathrm{l}$ serum-free RPMI medium in the upper chamber of 24-well transwell (Corning, NY, USA) and the lower chamber was filled with RPMI medium supplemented with 10\% FBS. For invasion assay, the only difference was that the upper chamber needed to be pre-coated with Matrigel (Corning). After $24 \mathrm{~h}$, the migrated or invaded cells on the lower surface were fixed and stained with crystal violet (Sinopharm Chemical Reagent, China) for $20 \mathrm{~min}$. Five random fields were selected to record cell numbers using an Olympus microscope (Olympus, Tokyo, Japan).

Bioinformatics analysis. The online software starBase (http://starbase.sysu.edu.cn) was used to predict the putative target genes of miR- 498 or TUG1 and analyze the binding sites between miR-498 and TUG1 or XBP1.

Luciferase reporter assay. Luciferase reporter assay was conducted to confirm the relationship between TUG1, miR-498, and XBP1. TUG1 wild-type (TUG1 wt) and TUG1 mutant (TUG1 mut) sequences containing miR-498 binding sites were inserted into the downstream of pmirGLO (Promega, Madison, WI, USA) to generate fusion vector, respectively. Afterward, the two fusion vectors were transfected into KYSE-150 and EC109 cells with miR-498 mimics or miR-NC, respectively. For XBP1, wild-type sequences of XBP1 3'UTR (XBP1 wt 3'UTR) or mutant sequences of XBP1 3'UTR (XBP1 mut 3'UTR) containing binding sites with miR-498 were also inserted into the downstream of pmirGLO vector to generate fusion vector. Then two fusion vectors together with miR-498 mimics or miR-NC were co-transfected into KYSE-150 and EC109 cells, respectively. After $48 \mathrm{~h}$, all cells were severally collected to measure luciferase activity by dual $\mathrm{Glo}^{\mathrm{m}}$ Luciferase Assay System (Promega).

Western blot analysis. Total proteins were extracted using Radio Immunoprecipitation Assay (RIPA) buffer (Thermo Fisher Scientific) from tissues or cells. Then, proteins were separated on $12 \%$ sodium dodecyl sulfate-polyacrylamide gel electrophoresis (SDS-PAGE) and transferred onto polyvinylidene fluoride (PVDF) membranes. Subsequently, the membranes were blocked with 5\% non-fat milk at room temperature for $2 \mathrm{~h}$ and incubated with the primary antibodies against XBP1 (ab37152; Abcam, Cambridge, MA, USA) or $\beta$-actin (ab8226; Abcam) at $4{ }^{\circ} \mathrm{C}$ overnight. The next day, the membranes were incubated with horseradish peroxidase-conjugated secondary antibody (Abcam) for $2 \mathrm{~h}$ after washed 3 times using PBS. Finally, the blots were visual- ized by using an enhanced chemiluminescence (ECL) kit (Beyotime, Shanghai, China) on the ChemiDoc MP imaging system (Bio-Rad).

Xenograft tumor mouse model analysis in vivo. The animal experiments were approved by the Animal Care and Use Committee of Gansu Provincial Hospital. The lentiviral vector (short hairpin RNA against TUG1 (sh-TUG1)) for stable TUG1 knockdown and control (sh-NC) were obtained from GenePharma (Shanghai). Male BALB/c nude mice (21-25 g weight, 6 weeks old) were purchased from the Shanghai Experimental Animal Center (Shanghai, China). EC109 cells transfected with sh-TUG1 or sh-NC were collected in PBS and subcutaneously injected into the groin of mice $\left(2 \times 10^{6}\right.$ cells/mouse, $\left.n=5\right)$. The mice were regularly fed and the tumor volume was measured every week. After 35 days, the mice were euthanized, and the tumor weight was detected. qRT-PCR and western blot assays were conducted to examine TUG1, miR-498 or XBP1 expression levels.

Statistical analysis. Statistical analyses were performed using SPSS 18.0 software (SPSS, Chicago, IL, USA). Differences between two groups were assessed using Student's t-test (two-tailed) or one-way analysis of variance (ANOVA) for multiple groups. The cumulative survival was calculated using the Kaplan-Meier method. The correlation was analyzed by using the Spearman rank correlation. Each experiment was performed at least three times. The results of the experiments were presented as the mean \pm standard deviation (SD). A p-value $<0.05$ was considered to be statistically significant.

\section{Results}

TUG1 was upregulated in ESCC tissues and was associated with the low survival of ESCC patients. To ascertain the role of TUG1 in ESCC, the expression of TUG1 in ESCC was measured by using qRT-PCR. Compared with normal tissues $(n=50)$, the expression of TUG1 was significantly increased in tumor tissues ( $n=50$, Figure 1A). Additionally, TUG1 was highly expressed in tumor tissues with lymph node metastasis $(n=21)$ than that in tumor tissues without lymph node metastasis ( $n=29$, Figure 1B). Besides, TUG1 expression was notably elevated at tumor node metastasis (TNM) stage III+IV $(n=24)$ than that at stage I+II $(n=26)$ of ESCC tumor tissues (Figure 1C). According to the average value of TUG1 expression in ESCC patients, patients were divided into a low TUG1 expression group $(\mathrm{n}=23)$ and a high TUG1 expression group ( $n=27)$. Kaplan-Meier curve presented a poor cumulative survival for ESCC patients with high TUG1 expression (Figure 1D). These data showed that TUG1 played a crucial role in ESCC and was associated with high mortality of ESCC patients.

TUG1 was highly expressed in ESCC cell lines and TUG1 knockdown inhibited proliferation, migration, and invasion but promoted apoptosis in ESCC cells. To investigate the potential function of TUG1, the expression of TUG1 in 

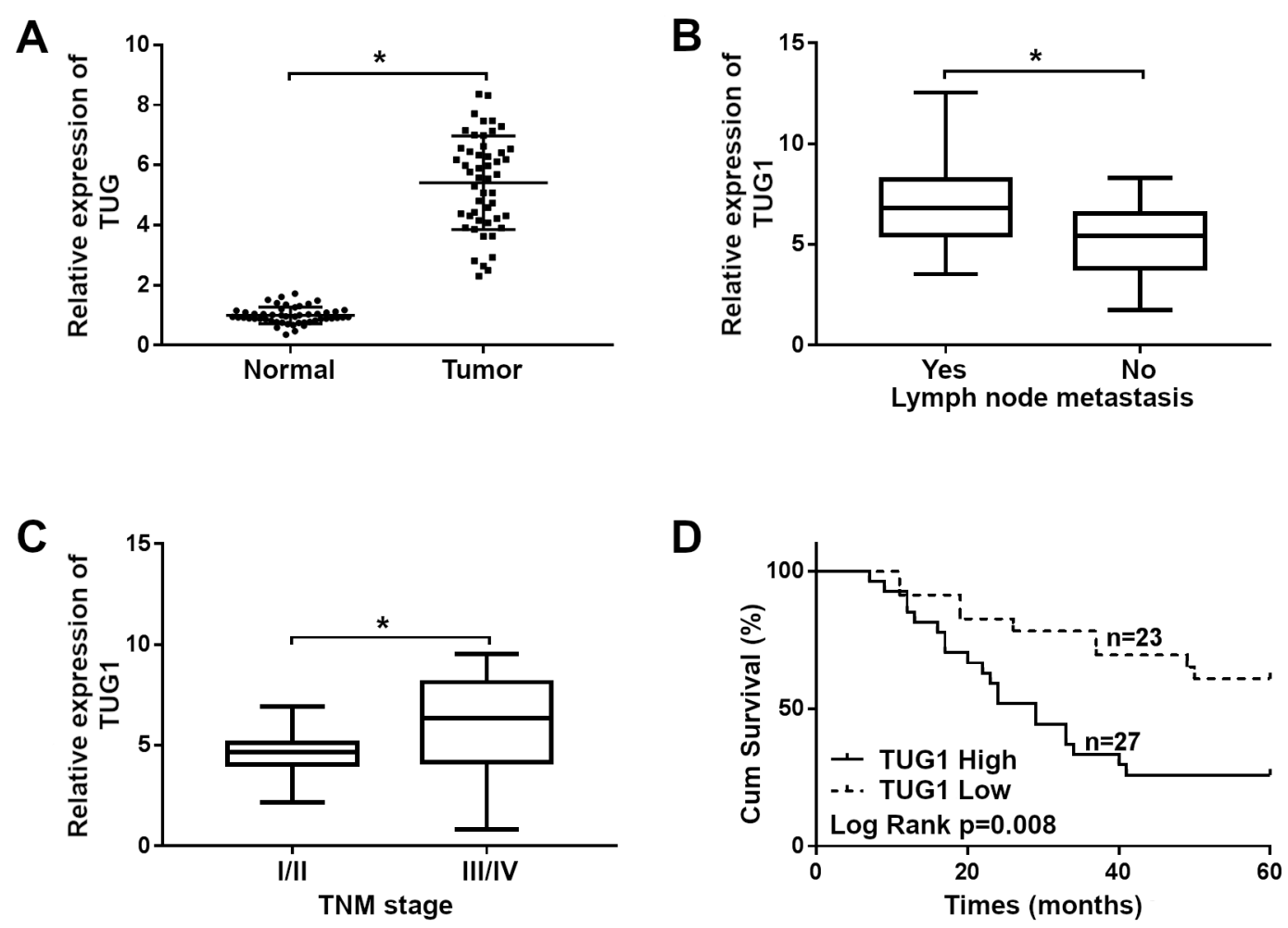

Figure 1. TUG1 was upregulated in ESCC tumor tissues and associated with a poor cumulative survival. A-C) $\mathrm{qRT}$-PCR analysis was performed to assess TUG1 expression level in ESCC tumor tissues $(n=50)$ and adjacent normal tissues $(n=50, A)$, tumor tissues of lymph node metastasis $(n=21)$ and non-metastasis $(n=29, B)$, TNM stage I+II tumor tissues $(n=26)$ and TNM stage III+IV tumor tissues $(n=24, C)$. D) The cumulative survival of ESCC patients with high TUG1 expression $(n=27)$ and low TUG1 expression $(n=23)$ was recorded. ${ }^{*} p<0.05$

ESCC cells was measured by qRT-PCR and the expression of TUG1 was knocked down for following functional analyses. As shown in Figure 2A, the expression of TUG1 was enhanced in all chosen ESCC cell lines, including KYSE-150, EC109, KYSE-450, TE-10, and KYSE-410 than that in human normal esophageal squamous cell line Het-1A. Then the efficiency of TUG1 knockdown was assessed by qRT-PCR and the result showed that TUG1 was prominently downregulated in KYSE-150 and EC109 cells transfected with si-TUG1\#1, si-TUG1\#2 and si-TUG1\#3. Obviously, the expression of TUG1 was reduced the most in the group of si-TUG1\#1 (Figure 2B). Hence si-TUG1\#1 group transfection was used for the next experiments. MTT assay showed that cell proliferation was pronouncedly inhibited in KYSE-150 (Figure 2C) and EC109 (Figure 2D) cells transfected with si-TUG1\#1 compared with si-NC for a sustained period of time. Flow cytometry indicated that si-TUG1\#1 transfection notably contributed to cell apoptosis rate in KYSE-150 and EC109 cells (Figure 2E). In addition, transwell assay identified that migration and invasion were markedly restrained once the cells were treated with si-TUG1\#1 (Figures 2F, 2G). All data suggested that TUG1 might function as an oncogene in ESCC cells.
miR-498 was a target of TUG1. To explore the underlying mechanism of TUG1 in ESCC development, the online software starBase was used to screen the possible target miRNAs and analyze the putative binding sites of TUG1. As shown in Figure 3A, there were several binding sites between TUG1 and miR-498. Moreover, the observation through luciferase reporter assay indicated that the luciferase activity was strongly weakened in the group of miR-498 mimics and TUG1 wt transfection, rather than that in other groups in KYSE-150 (Figure 3B) and EC109 cells (Figure 3C). Therefore, it could be considered that miR-498 was definitely a target of TUG1.

Next, we examined whether the expression of miR-498 was affected by the changes of TUG1 expression. The result claimed that the expression of miR-498 was advanced by TUG1 knockdown but inhibited by TUG1 overexpression both in KYSE-150 (Figure 3D) and EC109 (Figure 3E) cells compared with si-NC or vector. Additionally, qRT-PCR analysis alleged that miR-498 was steeply downregulated in tumor tissues than that in normal tissues (Figure 3F), and correlation analysis revealed that the expression of miR-498 was always negatively correlated with TUG1 (Figure 3G). The above analyses suggested that miR-498 was a target of TUG1 and its expression was regulated by TUG1. 

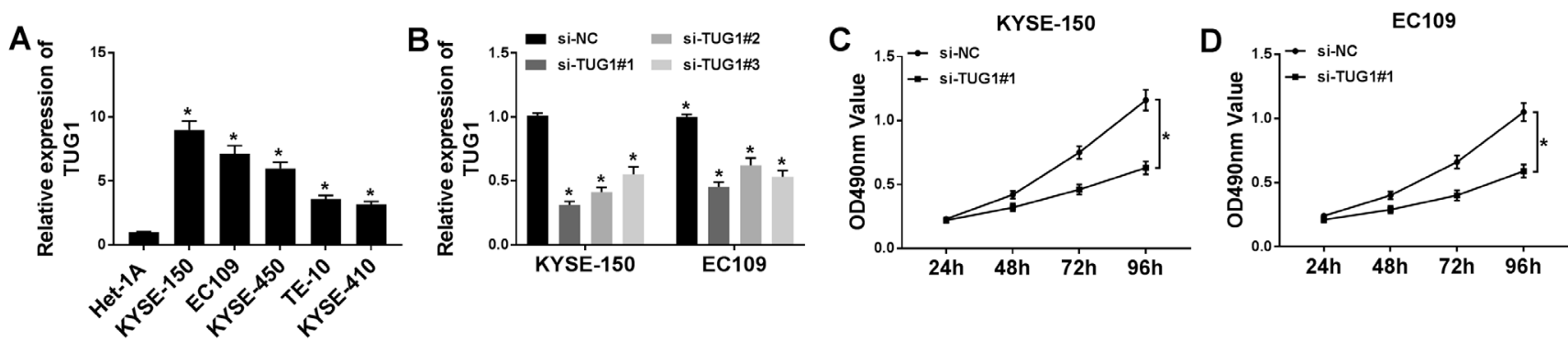
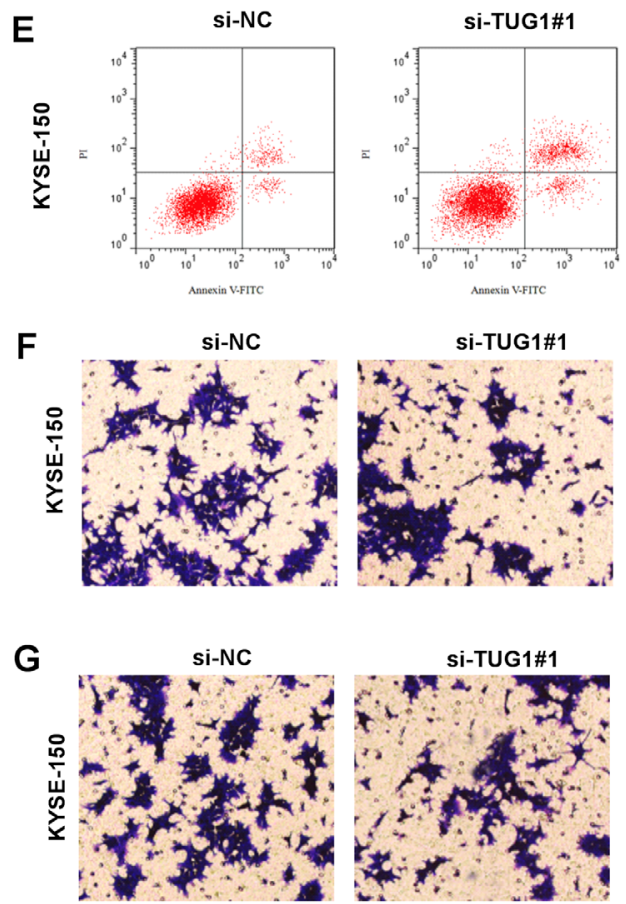
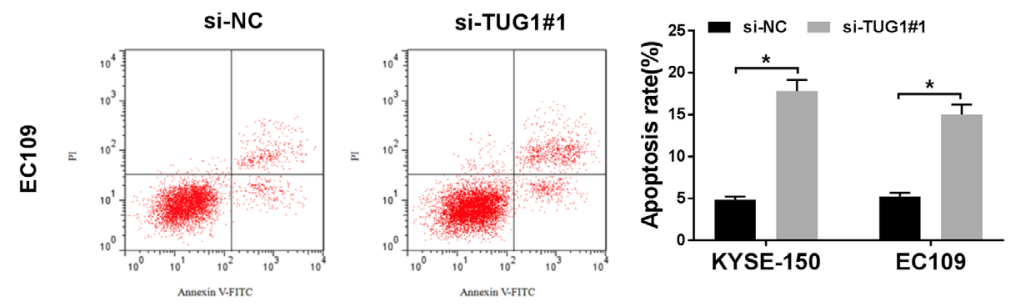

si-NC
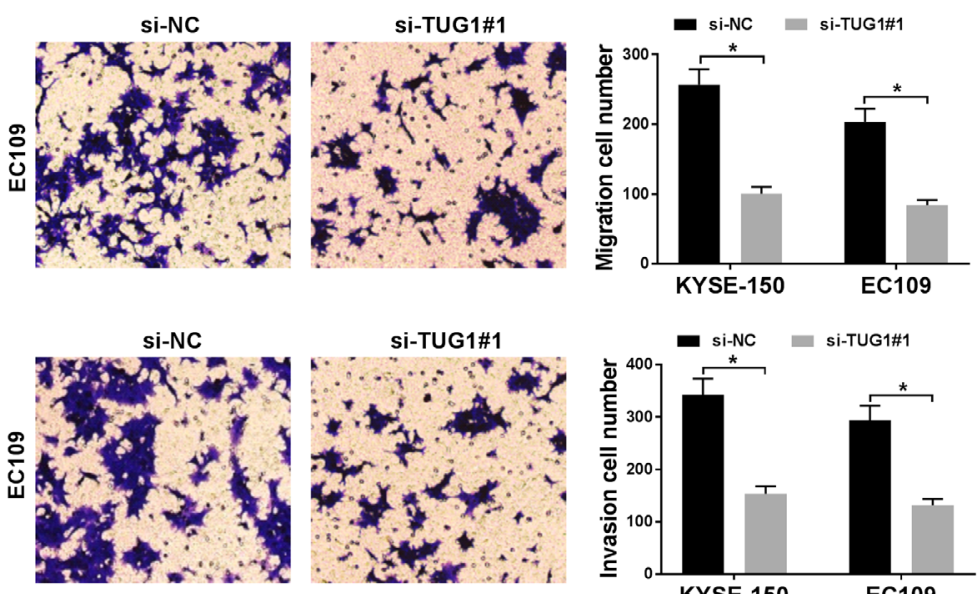

Figure 2. TUG1 knockdown inhibited proliferation, migration, and invasion but blocked apoptosis of ESCC cells. A) The expression of TUG1 in ESCC cell lines (KYSE-150, EC109, KYSE-450, TE-10, and KYSE-410) and normal cell line Het-1A was assessed by qRT-PCR. KYSE-150 and EC109 cells were transfected with si-TUG\#1, si-TUG\#2 and si-TUG\#3, si-NC as control. Then the efficiency of TUG1 B) knockdown; C, D) cell proliferation; E) apoptosis; F) migration and G) invasion were determined by qRT-PCR, MTT assay, flow cytometry assay, and transwell assay, respectively. ${ }^{\star} \mathrm{p}<0.05$.

TUG1 regulated proliferation, apoptosis, migration, and invasion of KYSE-150 and EC109 cells by targeting miR-498. To explore the potential mechanism by which TUG1 exerting its functions, KYSE-150 and EC109 cells were transfected with miR-498 mimics, miR-NC, si-TUG\#1+anti-miR-498 or si-TUG\#1+anti-NC. Firstly, qRT-PCR analysis elucidated that the expression of miR-498 quickly climbed in cells transfected with miR-498 mimics alone compared with miR-NC, while the expression of miR-498 was enormously declined in cells transfected with si-TUG1\#1+anti-498 compared with that in cells transfected with si-TUG1\#1+anti-NC (Figures 4A, 4B). MTT assay indicated that the cell viability was inhibited by miR-498 mimics, but cell viability inhibited by si-TUG1\#1+anti-NC was improved by si-TUG1\#1+antimiR-498 both in KYSE-150 (Figure 4C) and EC109 cells (Figure 4D). Flow cytometry manifested that the number of apoptotic cells was elevated in cells transfected with miR-498 mimics compared with miR-NC, while si-TUG1\#1+antimiR-498 transfection significantly dwindled the number of apoptotic cells promoted by si-TUG1\#1+anti-NC (Figure 4E). Transwell assay concluded that miR-498 mimics suppressed migration and invasion compared with miR-NC, whereas si-TUG1\#1+anti-miR-498 transfection accelerated migration and invasion depleted by si-TUG1\#1+anti-NC both in KYSE-150 and EC109 cells (Figures 4F, 4G). These data demonstrated that TUG1 exerted its role in cell proliferation, apoptosis, migration and invasion through directly targeting miR-498.

XBP1 was a target of miR-498 and TUG1 regulated the expression of XBP1 through targeting miR-498. To further distinguish the role of TUG1 in ESCC, we excavated a target of miR-498 and determined its expression. As we saw in 
A

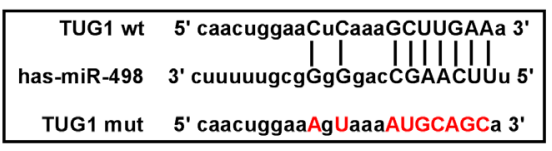

B

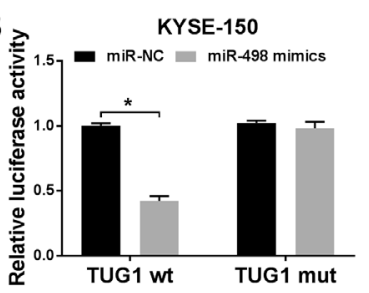

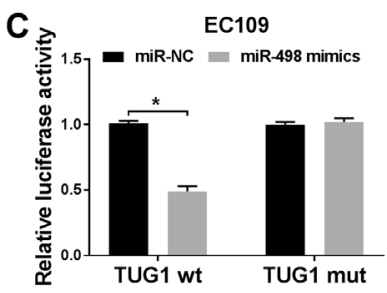

D

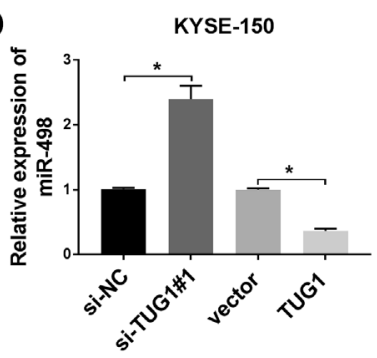

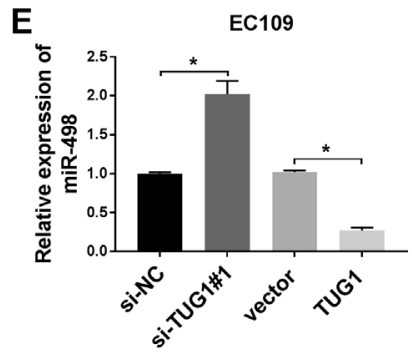

$\mathbf{F}$

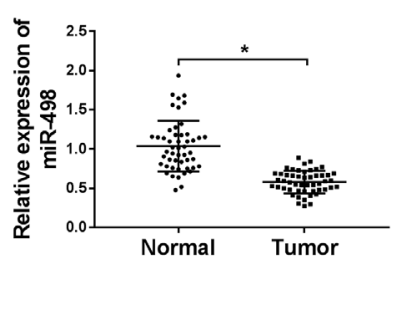

G응

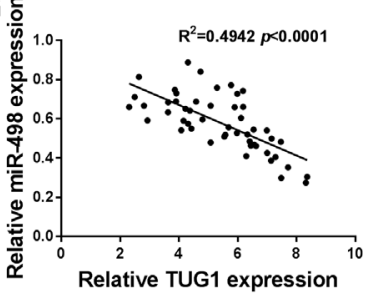

Figure 3. miR-498 was targeted by TUG1 and was downregulated in ESCC tissues and cells. A) The binding sites between TUG1 and miR-498 were shown. B, C) Luciferase reporter assay was conducted to verify the interaction between TUG1 and miR-498 in KYSE-150 and EC109 cells. D, E) The effects of TUG1 knockdown or overexpression on the expression of miR-498 were examined by qRT-PCR. F) The expression of miR-498 was decreased in tumor tissues compared with that in normal tissues of ESCC by qRT-PCR. G) The correlation between the expression of miR-498 and TUG1 was analyzed. ${ }^{\star} \mathrm{p}<0.05$
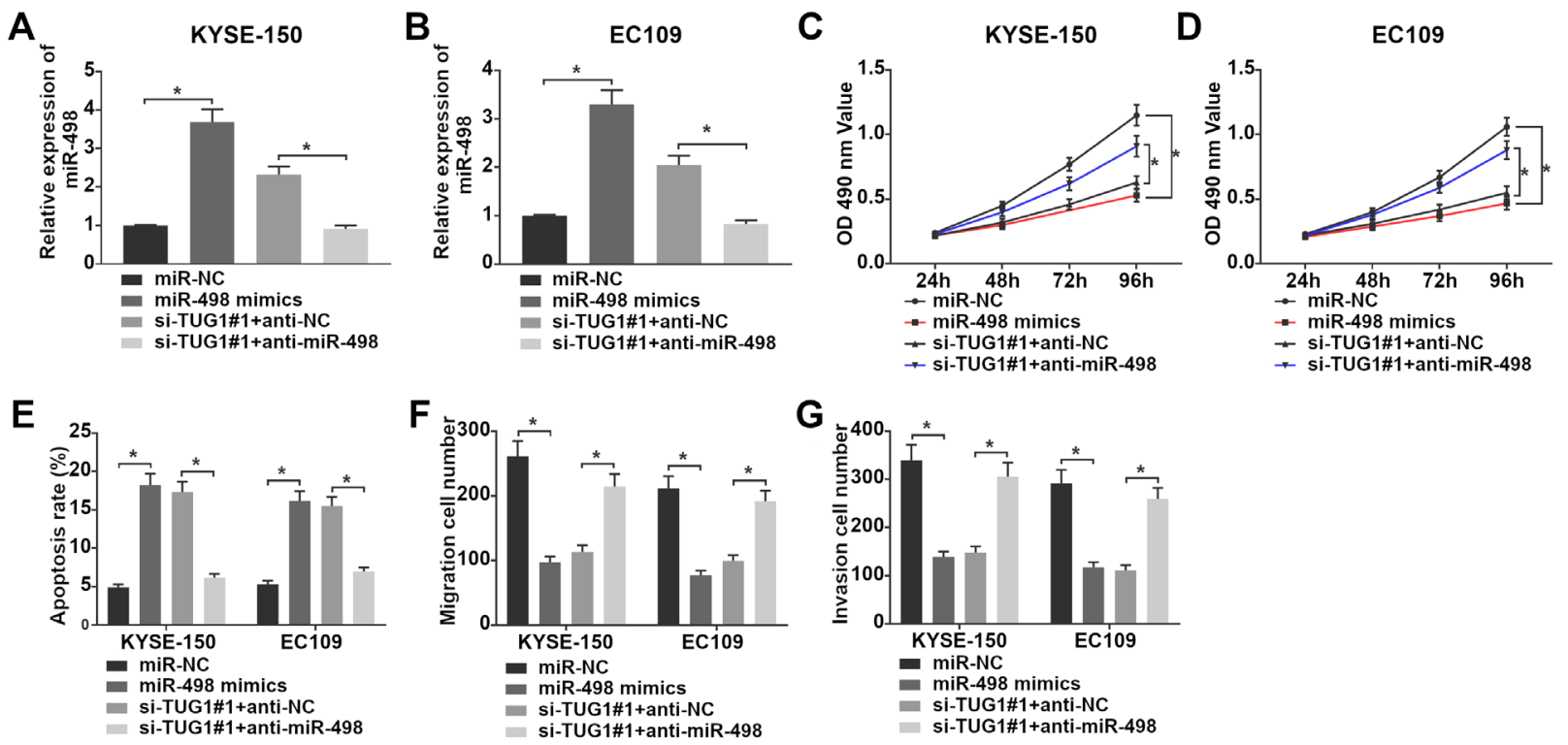

Figure 4. TUG1 regulated proliferation, apoptosis, migration, and invasion of KYSE-150 and EC109 cells by targeting miR-498. KYSE-150 and EC109 cells were transfected with miR-498 mimics, miR-NC, si-TUG\#1+anti-miR-498 or si-TUG1+anti-NC, respectively. Then the expression of miR-498 (A, B); proliferation (C, D); apoptosis (E); migration (F) and invasion (G) in KYSE-150 and EC109 were detected by qRT-PCR, MTT assay, flow cytometry assay, and transwell assay, respectively. ${ }^{*} \mathrm{p}<0.05$

Figure $5 \mathrm{~A}$, a special binding site existed between XBP1 and miR-498. Then, the sequences of XBP1 wt 3'UTR and the sequences of XBP1 mut 3'UTR were inserted into luciferase reporter vector pmirGLO, respectively. Luciferase activity was conspicuously decreased in both KYSE-150 and EC109 cells transfected with XBP1 wt 3'UTR sequences and miR-498 mimics, while the luciferase activity was recovered when cells were transfected with XBP1 wt 3'UTR sequences and miR-498 mimics+TUG1 wt. Whereas the luciferase activity in cells with XBP1 wt 3'UTR sequences and miR-498 mimics+TUG1 mut was still low. Here, miR-NC and XBP1 mut 3'UTR groups served as controls (Figures 5B, 5C). Next, the qRT-PCR analysis showed that the mRNA level of XBP1 expression was significantly reduced in cells transfected 
with miR-498 mimics compared with miR-NC (Figure 5D). Western blot analysis displayed that the protein level of XBP1 expression was also significantly decreased in the miR-498 mimics transfection group (Figure 5E). Afterward, KYSE-150 and EC109 cells were transfected with si-TUG\#1, si-NC, si-TUG\#1+anti-miR-498 or si-TUG\#1+anti-NC. The expression of XBP1 was weakened by si-TUG\#1 transfection compared with si-NC, but recovered by si-TUG\#1+antimiR-498 transfection compared with si-TUG\#1+antiNC from both mRNA level (Figure 5F) and protein level (Figure 5G). Eventually, we examined the expression of XBP1 in ESCC tumor tissues, and the result showed that XBP1 was obviously upregulated in tumor tissues compared with that in normal tissues (Figure 5H). Correlation analysis maintained that the expression of XBP1 was negatively correlated with miR-498 expression, but positively correlated with TUG1 expression (Figures 5I, 5J). The above data proved that XBP1 was a target of miR-498, and its expression was regulated by TUG1 through miR-498.

XBP1 overexpression reversed the effects on proliferation, apoptosis, migration, and invasion influenced by TUG1 knockdown or miR-498 mimics in KYSE-150 and EC109 cells. To further explore the mechanism of TUG1 in ESCC cells, KYSE-150 and EC109 cells were transfected with si-TUG\#1+pcDNA-XBP1, si-TUG\#1+pcDNA， miR-498 mimics+pcDNA-XBP1 or miR-498 mimics+pcDNA.
A
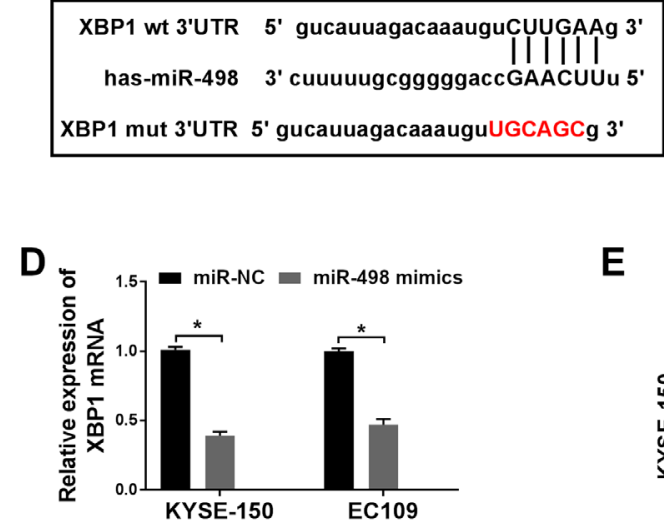

$\mathbf{F}$

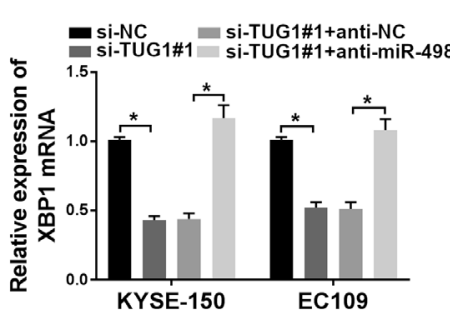

$\mathrm{H}$

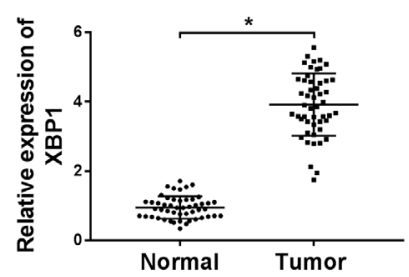

B

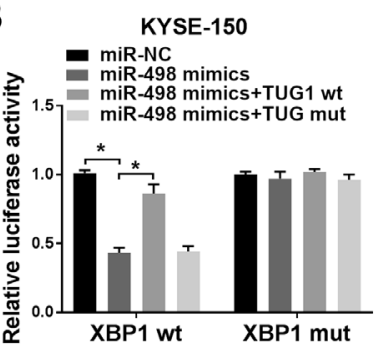

E

G

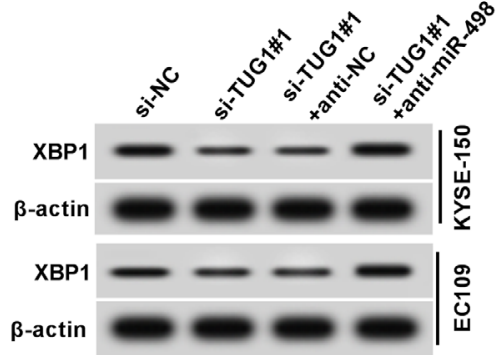

I
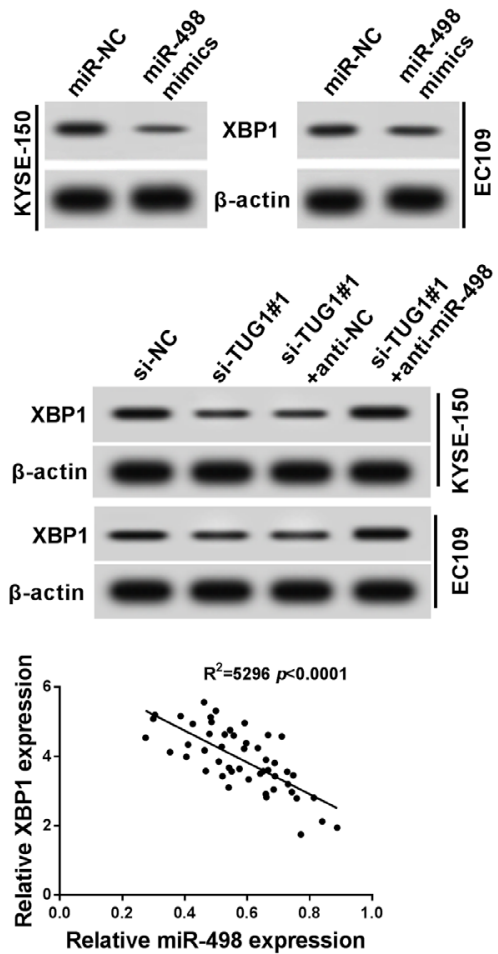

C
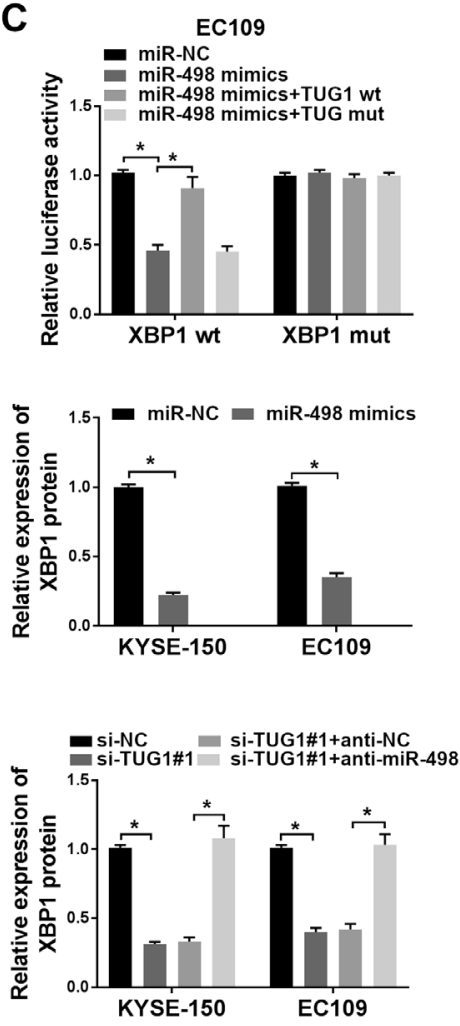

$\mathbf{J}$

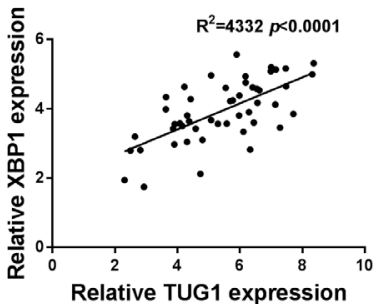

Figure 5. XBP1 was a target of miR-498. A) The binding sites between miR-498 and XBP1 3'UTR were exhibited. B, C) Luciferase reporter assay was employed to confirm the interaction between XBP1 and miR-498 in KYSE-150 and EC109 cells. D) The expression of XBP1 at the mRNA level was measured by qRT-PCR. E) The expression of XBP1 at the protein level was quantified by western blot. F, G) The expression of XBP1 was inhibited by TUG1 knockdown but reversed by miR-498 knockdown meanwhile in KYSE-150 and EC109 cells at mRNA level (F) and protein level (G). H) XBP1 was upregulated in tumor tissues compared with that in normal tissues. I, J) Correlation analyses revealed that XBP1 expression was negatively correlated with miR-498 expression (I) but was positively correlated with TUG1 expression (J). ${ }^{*} \mathbf{p}<0.05$ 
Initially, the protein level of XBP1 was monitored, and western blot analysis showed that the expression of XBP1 was notably enhanced in si-TUG\#1+pcDNA-XBP1 transfection compared with si-TUG\#1+pcDNA. Likewise, the expression of XBP1 was markedly strengthened in miR-498 mimics+pcDNA-XBP1 transfection compared with miR-498 mimics+pcDNA (Figure 6A). For functional analyses, MTT assay stated that si-TUG\#1+pcDNA-XBP1 or miR-498 mimics+pcDNA-XBP1 transfection reversed cell proliferation inhibited by si-TUG\#1+pcDNA or miR-498 mimics+pcDNA transfection in KYSE-150 and EC109 cells, respectively (Figures 6B, 6C). Flow cytometry expounded that cell apoptosis rate was significantly declined in cells transfected with si-TUG\#1+pcDNA-XBP1 compared with si-TUG\# $1+$ pcDNA, and also declined in cells transfected with miR-498 mimics+pcDNA-XBP1 compared with miR-498 mimics+pcDNA (Figure 6D). Transwell assay pointed out that pcDNA-XBP1 could recover the number of migrated and invaded cells suppressed by si-TUG1\#1 or miR-498 mimics (Figures 6E, 6F). These data claimed that TUG1 regulated proliferation, apoptosis, migration, and invasion by regulating the expression of XBP1 via modulating miR- 498 .

TUG1 knockdown inhibited tumor growth by downregulating XBP1 through targeting miR-498 in vivo. To investigate the effect on tumor formation of TUG1 in vivo, a xenograft tumor mouse model was established. EC109 cells transfected with sh-TUG1 or sh-NC were subcutaneously injected into nude mice. As presented in Figure 7A, the TUG1 knockdown significantly blocked tumor volume. After 35 days, mice were euthanized and the weight was notably diminished in mice inoculated with sh-TUG1 compared with sh-NC (Figure 7B). Additionally, the qRT-PCR analysis showed that TUG1 expression was prominently lower in tumor tissues from the sh-TUG1 group than the sh-NC group (Figure 7C). The expression of miR-498 was obviously enhanced in tumor tissues from the sh-TUG1 group than the sh-NC group (Figure 7D). Meanwhile, the expression of XBP1 was strikingly attenuated in tumor tissues from the sh-TUG1 group than the sh-NC group from both mRNA level and protein level (Figures 7E, 7F). Thus, it was concluded that the TUG1 knockdown impeded tumor growth by depleting XBP1 expression by enhancing miR-498 expression in vivo.

\section{Discussion}

ESCC accounts for about $90 \%$ of esophageal cancers (456,000 cases) each year. With high mortality, ESCC is a complex disease with many causes that differs by histologic type and the population [28]. Hence, the research on the potential mechanism of ESCC is valuable and essential. In the present study, TUG1 was upregulated in ESCC tissues compared with normal adjacent tissues, and TUG1 overexpression was correlated with TNM stage, lymph node metastasis, and low cumulative survival. Functional analyses demonstrated that TUG1 was upregulated in ESCC cell lines and TUG1 knockdown inhibited proliferation, migration, and invasion but promoted apoptosis in ESCC cells. It was confirmed that miR-498 was a target of TUG1 and XBP1 was a target of miR-498 here. Functional study manifested that TUG1 regulated proliferation, apoptosis, migration, and
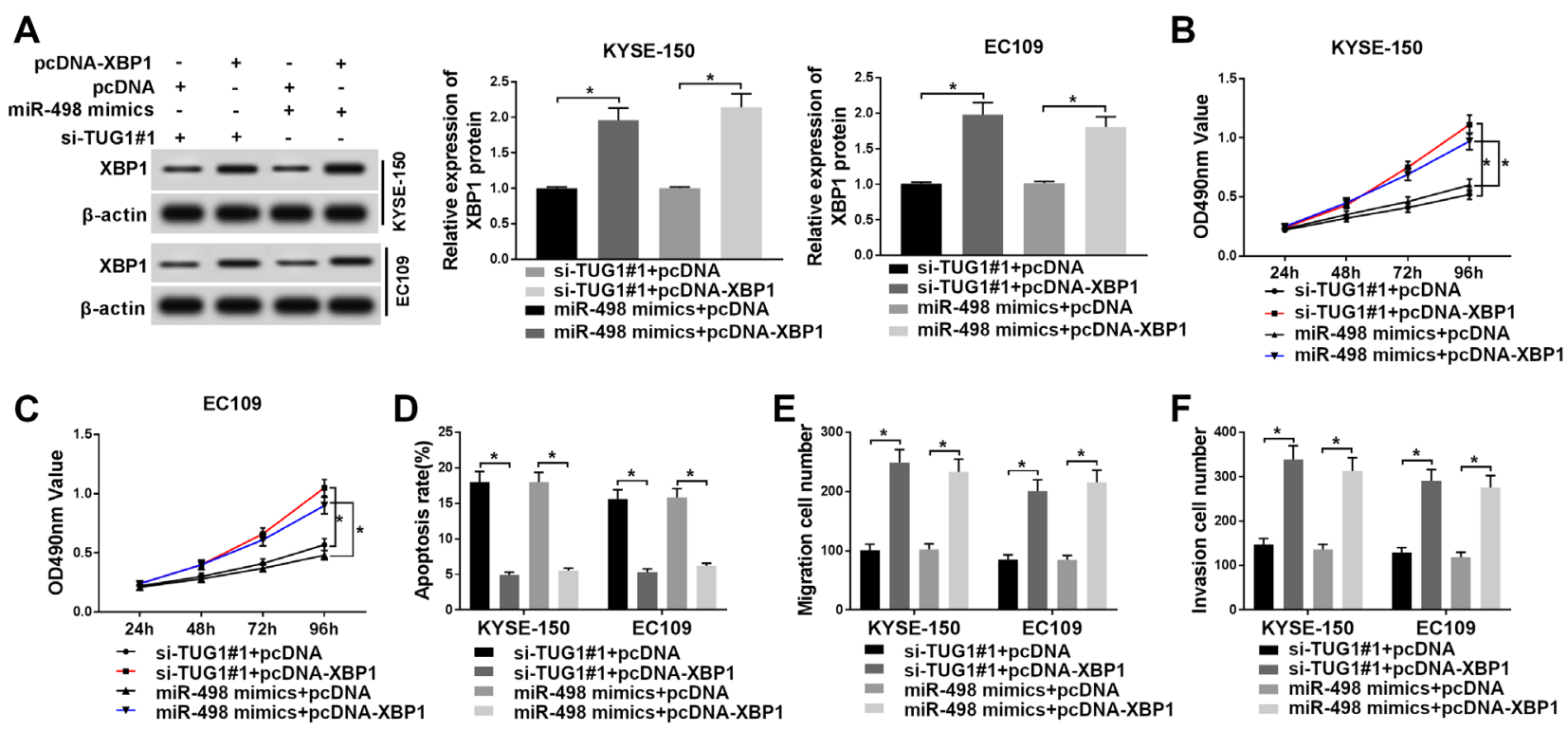

Figure 6. XBP1 overexpression reversed the effects on proliferation, apoptosis, migration, and invasion caused by TUG1 knockdown or miR-498 enrichment. KYSE-150 and EC109 cells were transfected with si-TUG\# 1+pcDNA-XBP1, si-TUG\#1+pcDNA, miR-498 mimics+pcDNA-XBP1 or miR-498 mimics+pcDNA. Then, A) the expression of XBP1 was assessed by western blot. B, C) Cell proliferation was detected by MTT assay. D) Cell apoptosis was checked by flow cytometry. E, F) Cell migration and invasion were ascertained by transwell. ${ }^{*} \mathrm{p}<0.05$ 
A
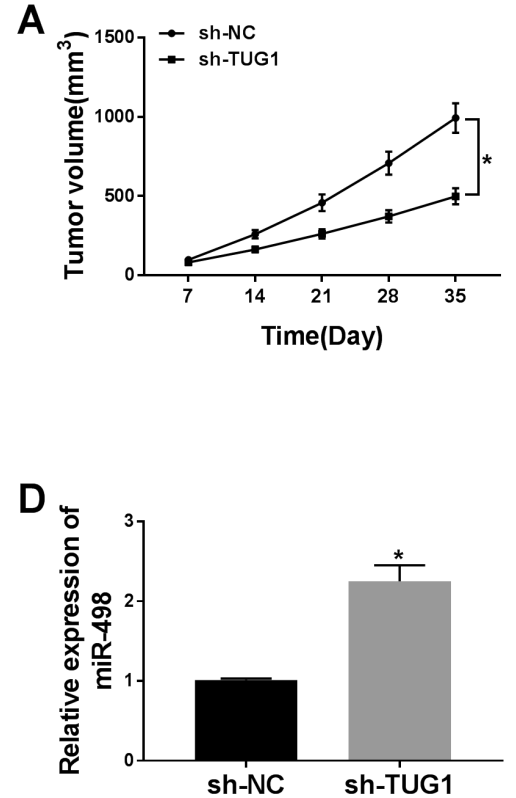

B
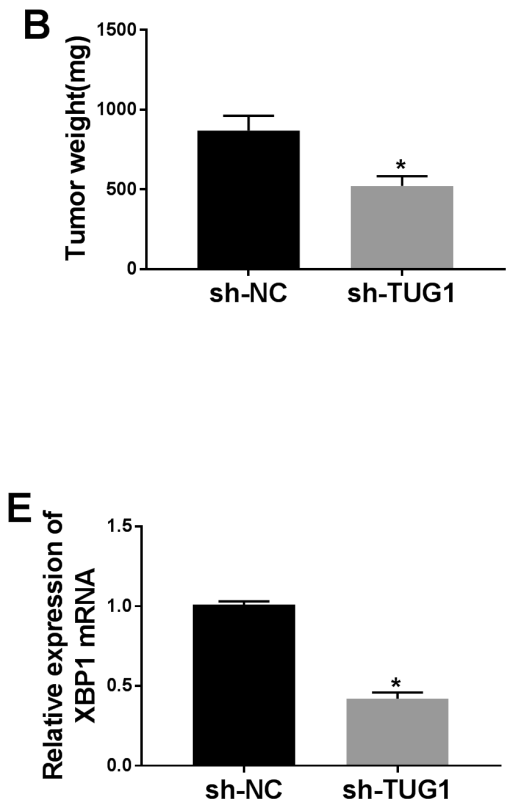
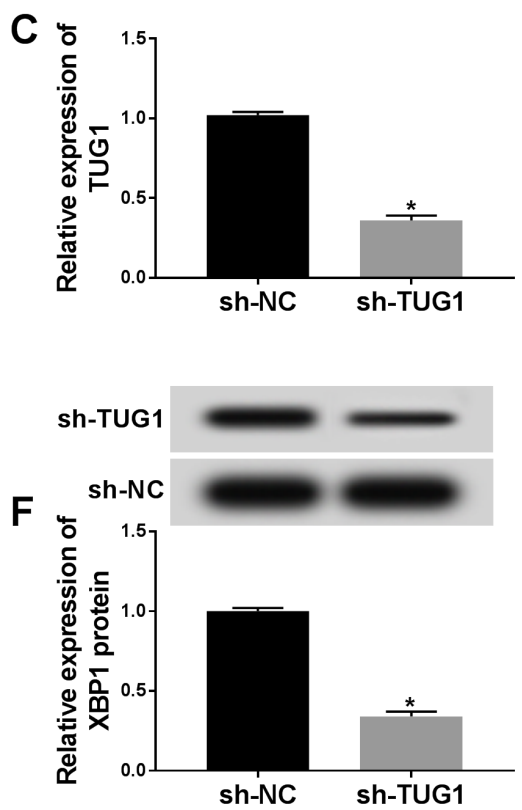

Figure 7. TUG1 knockdown suppressed tumor growth in vivo. A) The tumor volume was recorded every week. B) TUG1 knockdown reduced the tumor weight. C) TUG1 was downregulated in mice tumor tissues injected with sh-TUG1, while D) miR-498 was upregulated. The expression of XBP1 was enhanced in mice tumor tissues both on the mRNA level $(\mathrm{E})$ and protein level $(\mathrm{F}) .{ }^{*} \mathrm{p}<0.05$.

invasion by upregulating the expression of XBP1 via targeting miR-498 in vitro. Moreover, knockdown of TUG1 attenuated tumor growth in vivo.

TUG1 acting as a carcinoma gene has been reported in numerous studies. For example, TUG1 promoted DDP resistance in TE-1 and TE-1/DDP cells by ameliorating cell proliferation, suppressing cell apoptosis, and elevating the expression of the classical multidrug resistance-related proteins in ESCC [29]. TUG1 was significantly overexpressed in ESCC tissues compared with paired adjacent normal tissues and silencing of TUG1 inhibited proliferation and migration but blocked the progression of the cell cycle [30]. TUG1 was upregulated in DDP-resistant ESCC tissues and cells, and high TUG1 expression was associated with the poor prognosis of ESCC patients [31]. Consistent with these findings, our study also found that TUG1 was upregulated in ESCC tissues and cells with, and high TUG1 expression was linked to low cumulative survival, suggesting that it indeed served as an oncogene in ESCC. However, several reports insisted that TUG1 was downregulated in tumor tissues and cells, and functioned as a tumor suppressor in breast cancer [32] and glioma [33]. This is mainly due to the different expression patterns of TUG1 in different types of cancer.

It is well known that lncRNAs can act as ceRNAs of specific miRNAs to regulate miRNA and downstream target mRNA expression. Here, the interaction between miR-498 and TUG1 was proved. A previous study characterized that miR-498 expression was significantly diminished in ESCC tissues and cells, and miR-498 served as a tumor suppressor, leading to the reduction of cell proliferation, barrier penetration, and colony formation [34]. In agreement with this research, we observed that the expression of miR-498 was also declined in ESCC tissues, and miR-498 knockdown had the ability to rescue the effects caused by TUG1 knockdown in ESCC cells. Therefore, miR-498 may act as a tumor suppressor in ESCC.

It was confirmed that XBP1 was targeted by miR-498 in this study. XBP1, found in the early 1990s, was identified as a key regulator of the expression of major histocompatibility complex class II genes in B cells $[35,36]$. XBP1 was reported as a carcinogenic factor in diverse types of cancer, such as triple-negative breast cancer [37], oral squamous cell carcinoma [38], and hepatocellular carcinoma [39]. Particularly, a previous study reported that XBP1 was markedly overexpressed in ESCC cell lines and clinical samples, and it facilitated proliferation and invasion by inducing the expression of MMP-9 in ESCC cells [26]. Consistent with these early researches, XBP1 in this paper was overexpressed in ESCC tissues and contributed to tumor growth and metastasis. Thus, XBP1 may promote tumor progression in ESCC.

In summary, we found that TUG1 was upregulated in ESCC tissues and cell lines. All data indicated its oncogenic property in ESCC tumorigenesis. Collectively, our current study revealed a novel action mechanism of TUG1 in ESCC that TUG1 promoted tumor growth and metastasis by regulating XBP1 via competitively binding to miR-498. TUG1 may serve as a candidate therapeutic target for ESCC therapy. 


\section{References}

[1] ENZINGER PC, MAYER RJ. Esophageal cancer. N Engl J Med 2003; 349: 2241-2252. https://doi.org/10.1056/NEJMra035010

[2] GUO Y, CHEN Z, ZHANG L, ZHOU F, SHI S et al. Distinctive MicroRNA profiles relating to patient survival in esophageal squamous cell carcinoma. Cancer Res 2008; 68: 26-33. https://doi.org/10.1158/0008-5472.CAN-06-4418

[3] JIANG L, WANG W, LI G, SUN C, REN Z et al. High TUG1 expression is associated with chemotherapy resistance and poor prognosis in esophageal squamous cell carcinoma. Cancer Chemother Pharmacol 2016; 78: 333-339. https:// doi.org/10.1007/s00280-016-3066-y

[4] MATHÉ EA, NGUYEN GH, BOWMAN ED, ZHAO Y, BUDHU A et al. MicroRNA expression in squamous cell carcinoma and adenocarcinoma of the esophagus: associations with survival. Clin Cancer Res 2009; 15: 6192-6200. https://doi.org/10.1158/1078-0432.CCR-09-1467

[5] MATSUSHIMA K, ISOMOTO H, YAMAGUCHI N, INOUE N, MACHIDA $\mathrm{H}$ et al. MiRNA-205 modulates cellular invasion and migration via regulating zinc finger E-box binding homeobox 2 expression in esophageal squamous cell carcinoma cells. J Transl Med 2011; 9: 30. https://doi. org/10.1186/1479-5876-9-30

[6] QI P, DU X. The long non-coding RNAs, a new cancer diagnostic and therapeutic gold mine. Mod Pathol 2013; 26: 155-165. https://doi.org/10.1038/modpathol.2012.160

[7] WILUSZ JE, SUNWOO H, SPECTOR DL. Long noncoding RNAs: functional surprises from the RNA world. Genes Dev 2009; 23: 1494-1504. https://doi.org/10.1101/gad.1800909

[8] PRENSNER JR, CHINNAIYAN AM. The Emergence of lncRNAs in Cancer Biology. Cancer Discov 2011; 1: 391-407. https://doi.org/10.1158/2159-8290.CD-11-0209

[9] QIU MT, HU J-W, YIN R, XU L. Long noncoding RNA: an emerging paradigm of cancer research. Tumour Biol 2013; 34: 613-620. https://doi.org/10.1007/s13277-013-0658-6

[10] YOUNG TL, MATSUDA T, CEPKO CL. The Noncoding RNA Taurine Upregulated Gene 1 Is Required for Differentiation of the Murine Retina. Curr Biol 2005; 15: 501-512. https://doi.org/10.1016/j.cub.2005.02.027

[11] ZHANG EB, YIN DD, SUN M, KONG R, LIU XH et al. P53regulated long non-coding RNA TUG1 affects cell proliferation in human non-small cell lung cancer, partly through epigenetically regulating HOXB7 expression. Cell Death Dis 2014; 5: e1243. https://doi.org/10.1038/cddis.2014.201

[12] JI TT, HUANG X, JIN J, PAN SH, ZHUGE XJ. Inhibition of long non-coding RNA TUG1 on gastric cancer cell transference and invasion through regulating and controlling the expression of miR-144/c-Met axis. Asian Pac J Trop Med 2016; 9: 508-512. https://doi.org/10.1016/j. apjtm.2016.03.026

[13] SUN J, DING C, YANG Z, LIU T, ZHANG X et al. The long non-coding RNA TUG1 indicates a poor prognosis for colorectal cancer and promotes metastasis by affecting epithelial-mesenchymal transition. J Transl Med 2016; 14: 42. https://doi.org/10.1186/s12967-016-0786-Z
[14] HUANG MD, CHEN WM, QI FZ, XIA R, SUN M et al. Long non-coding RNA TUG1 is up-regulated in hepatocellular carcinoma and promotes cell growth and apoptosis by epigenetically silencing of KLF2. Mol Cancer 2015; 14: 165. https://doi.org/10.1186/s12943-015-0431-0

[15] HAN Y, LIU Y, GUI Y, CAI Z. Long intergenic non-coding RNA TUG1 is overexpressed in urothelial carcinoma of the bladder. J Surg Oncol 2013; 107: 555-559. https://doi. org/10.1002/jso.23264

[16] ZHANG X, XU X, GE G, ZANG X, SHAO M, ZOU S et al. miR-498 inhibits the growth and metastasis of liver cancer by targeting ZEB2. Oncol Rep 2019; 41: 1638-1648. https://doi. org/10.3892/or.2018.6948

[17] RUPAIMOOLE R, CALIN GA, LOPEZ-BERESTEIN G, SOOD AK. miRNA Deregulation in Cancer Cells and the Tumor Microenvironment. Cancer Discov 2016; 6: 235-246. https://doi.org/10.1158/2159-8290.CD-15-0893

[18] HIYOSHI Y, KAMOHARA H, KARASHIMA R, SATO N, IMAMURA $Y$ et al. MicroRNA-21 Regulates the Proliferation and Invasion in Esophageal Squamous Cell Carcinoma. Clin Cancer Res 2009; 15: 1915-1922. https://doi. org/10.1158/1078-0432.CCR-08-2545

[19] GUO H, WANG K, XIONG G, HU H, WANG D et al. A functional varient in microRNA-146a is associated with risk of esophageal squamous cell carcinoma in Chinese Han. Fam Cancer 2010; 9: 599-603. https://doi.org/10.1007/s10689010-9370-5

[20] YUAN Y, ZENG ZY, LIU XH, GONG DJ, TAO J et al. MicroRNA-203 inhibits cell proliferation by repressing $\triangle \mathrm{Np} 63$ expression in human esophageal squamous cell carcinoma. BMC Cancer 2011; 11: 57. https://doi.org/10.1186/14712407-11-57

[21] WANG M, ZHANG Q, WANG J, ZHAI Y. MicroRNA-498 is downregulated in non-small cell lung cancer and correlates with tumor progression. J Cancer Res Ther 2015; 11 : C107-111. https://doi.org/10.4103/0973-1482.163859

[22] CONG J, LIU R, WANG X, WANG J, WANG H et al. Low miR-498 expression levels are associated with poor prognosis in ovarian cancer. Eur Rev Med Pharmacol Sci 2015; 19: 4762-4765.

[23] GOPALAN V, SMITH RA, LAM AK. Downregulation of microRNA-498 in colorectal cancers and its cellular effects. Exp Cell Res 2015; 330: 423-428. https://doi.org/10.1016/j. yexcr.2014.08.006

[24] ANDRES SA, WITTLIFF JL. Relationships of ESR1 and XBP1 expression in human breast carcinoma and stromal cells isolated by laser capture microdissection compared to intact breast cancer tissue. Endocrine 2011; 40: 212-221. https://doi.org/10.1007/s12020-011-9522-x

[25] SUN Y, JIANG F, PAN Y, CHEN X, CHEN J et al. XBP1 promotes tumor invasion and is associated with poor prognosis in oral squamous cell carcinoma. Oncol Rep 2018; 40: 988-998. https://doi.org/10.3892/or.2018.6498

[26] XIA T, TONG S, FAN K, ZHAI W, FANG B et al. XBP1 induces MMP-9 expression to promote proliferation and invasion in human esophageal squamous cell carcinoma. Am J Cancer Res 2016; 6: 2031-2040. 
[27] LI H, CHEN X, GAO Y, WU J, ZENG F et al. XBP1 induces snail expression to promote epithelial- to-mesenchymal transition and invasion of breast cancer cells. Cell Signal 2015; 27: 82-89. https://doi.org/10.1016/j.cellsig.2014.09.018

[28] ABNET CC, ARNOLD M, WEI WQ. Epidemiology of Esophageal Squamous Cell Carcinoma. Gastroenterology 2018; 154: 360-373. https://doi.org/10.1053/j.gastro.2017.08.023

[29] ZHANG Z, XIONG R, LI C, XU M, GUO M. LncRNA TUG1 promotes cisplatin resistance in esophageal squamous cell carcinoma cells by regulating Nrf2. Acta Biochim Biophys Sin (Shanghai) 2019; 51: 826-833. https://doi.org/10.1093/ abbs/gmz069

[30] XU Y, WANG J, QIU M, XU L, LI M et al. Upregulation of the long noncoding RNA TUG1 promotes proliferation and migration of esophageal squamous cell carcinoma. Tumour Biol 2015; 36: 1643-1651. https://doi.org/10.1007/s13277014-2763-6

[31] XU C, GUO Y, LIU H, CHEN G, YAN Y et al. TUG1 confers cisplatin resistance in esophageal squamous cell carcinoma by epigenetically suppressing PDCD4 expression via EZH2. Cell Biosci 2018; 8: 61. https://doi.org/10.1186/s13578-0180260-0

[32] FAN S, YANG Z, KE Z, HUANG K, LIU N et al. Downregulation of the long non-coding RNA TUG1 is associated with cell proliferation, migration, and invasion in breast cancer. Biomed Pharmacother 2017; 95: 1636-1643. https://doi. org/10.1016/j.biopha.2017.09.076
[33] LI J, ZHANG M, AN G, MA Q. LncRNA TUG1 acts as a tumor suppressor in human glioma by promoting cell apoptosis. Exp Biol Med (Maywood) 2016; 241: 644-649. https:// doi.org/10.1177/1535370215622708

[34] ISLAM F, GOPALAN V, LAW S, TANG JC, CHAN KW, et al. MiR-498 in esophageal squamous cell carcinoma: clinicopathological impacts and functional interactions. Hum Pathol 2017; 62: 141-151. https://doi.org/10.1016/j.humpath.2017.01.014

[35] LIOU H, BOOTHBY M, FINN P, DAVIDON R, NABAVI $\mathrm{N}$ et al. A new member of the leucine zipper class of proteins that binds to the HLA DR alpha promoter. Science 1990; 247: 1581-1584. https://doi.org/10.1126/science.2321018

[36] HE Y, SUN S, SHA H, LIU Z, YANG L et al. Emerging Roles for XBP1, a sUPeR Transcription Factor. Gene Expr 2010; 15: 13-25. https://doi.org/10.3727/105221610x12819686555051

[37] CHEN X, ILIOPOULOS D, ZHANG Q, TANG Q, GLIMCHER LH et al. XBP1 Promotes Triple Negative Breast Cancer By Controlling the HIF1 a Pathway. Nature 2014; 508: 103-107. https://doi.org/10.1038/nature13119

[38] SUN Y, JIANG F, PAN Y, CHEN X, CHEN J et al. XBP1 promotes tumor invasion and is associated with poor prognosis in oral squamous cell carcinoma. Oncol Rep 2018; 40: 988-998. https://doi.org/10.3892/or.2018.6498

[39] ZHOU T, LV X, GUO X, RUAN B, LIU D et al. RACK1 modulates apoptosis induced by sorafenib in HCC cells by interfering with the IRE1/XBP1 axis. Oncol Rep 2015; 33: 3006-3014. https://doi.org/10.3892/or.2015.3920 